\title{
On the $\Phi$-Invariant of Two Quadrics
}

\author{
By T. Scots
}

(Received 31st March 1950. Read 5th May 1950.)

\section{Introduction.}

The geometry associated with the five invariants of two quadrics is well known.' There appears to be an omission, however, in the treatment of the $\Phi$-invariant. Salmon gives the vanishing of $\Phi$ merely as a necessary condition for the possibility of the construction of a tetrahedron self-conjugate for one of the quadrics and having its six edges tangential to the other. ${ }^{2}$ Sommerville proves its sufficiency, and shows that this condition is poristic, giving rise to two systems of $\infty^{1}$ tetrahedra of the requisite type. ${ }^{3}$ No investigation appears to have been made of the locus of the vertices of the $\infty^{1}$ tetrahedra of each system and the dual problem regarding the nature of the developable surface arising from their $\propto^{1}$ faces.

The purpose of this paper is to derive the equations of these constructs, showing the curve to be of order eight, and the developable to be of class eight. The treatment throughout is based on the Clebsch-Aronhold symbolism, ${ }^{4}$ viz.,

$$
\begin{aligned}
& f \equiv a_{x}^{2}, \quad f^{\prime} \equiv b_{x}^{2} . \\
& \Sigma \equiv u_{a}^{2}, \quad \Sigma^{\prime} \equiv u_{\beta}^{2}, \quad\left(a \equiv a a^{\prime} a^{\prime \prime}, \beta \equiv b b^{\prime} b^{\prime \prime}\right) \text {. } \\
& \Pi \equiv(A p)^{2}, \quad \Pi^{\prime} \equiv(B p)^{2}, \quad\left(A \equiv a a^{\prime}, \quad B \equiv b b^{\prime}\right) \text {. } \\
& k \equiv(A \beta x)^{2}, \quad k^{\prime} \equiv(B a x)^{2}, \quad\left[(A \beta x) \equiv \dot{a}_{\beta} \dot{a}_{x}^{\prime} \equiv \bar{a}_{\beta} \bar{a}_{x}^{\prime}\right] . \\
& \chi \equiv(A b u)^{2}, \quad \chi^{\prime} \equiv(B a u)^{2} .
\end{aligned}
$$

The five invariants are then represented as follows:-

1 (1) Salmon, A Treatise on the Analytic Geometry of Three Dimensions (revised by Rogers), Ch. IX.

(2) Sommerville, Analytical Geometry of Three Dimensions, Ch. XV.

(3) H. W. Turnbull, "Some Geometrical Interpretations of the Concomitants of Two Quadrics," Proc. Cambridge Phil. Soc., XIX (1919), 196.206.

2 (1) p. 204, §201.

3 (2) p. 312, $\$ 15.22$.

(3) p. 198, $\$$. $4,5$. 


$$
\Delta \equiv \stackrel{2}{a_{a}}, \Theta \equiv b_{a}^{2}, \Phi \equiv(A B)^{2}, \Theta^{\prime} \equiv a_{\beta}^{2}, \Delta^{\prime} \equiv b_{\beta}^{2} .
$$

In the following account it will be proved that, when $\Phi$ vanishes, the developable containing the faces of one system of the abovementioned tetrahedra is formed by the $\infty^{1}$ planes common to the quadric and quartic envelopes

$$
\chi=0, \quad \Delta \Sigma^{\prime 2}-16 \Theta^{\prime} \Sigma \Sigma^{\prime}+12 \Delta^{\prime} \Sigma \chi^{\prime}=0
$$

while the locus of the $\infty^{1}$ vertices is the curve of intersection of the quadric and quartic surfaces

$$
k=0, \quad \Delta f^{\prime 2}-16 \Theta f f^{\prime}+8 f k^{\prime}=0 .
$$

The equations of the developable and curve associated with the second system of tetrahedra will also be given in similar form.

2. The vanishing of $\Phi$ as a necessary condition.

Let $P Q R S$ be a tetrahedron self-congugate for $f^{\prime}$ and having its. six edges touching $f$. If $u$ represents the plane $Q R S$ then the conic envelope of lines in $u$ belonging to $I I$ is given by

$$
(A u v)^{2}=0 \text { (v current). }
$$

The triangle $Q R S$ therefore circumscribes the conic $(A u v)^{2}$ and is selfconjugate for the section of $f^{\prime}$ by $u$. That is, $(A u v)^{2}$ is inpolar to $f^{\prime}$, and so $u$ satisfies the equation

$$
(A b u)^{2}=0 .
$$

Thus, the plane $Q R S$ touches the quadric envelope $\chi$. Similarly, all four faces of the tetrahedron $P Q R S$ touch $\chi$, and since the tetrahedron circumscribes $\chi$ and is self-conjugate for $f^{\prime}, \chi$ is inpolar to $f^{\prime}$, and so $\left(A b b^{\prime}\right)^{2}=0$.

Therefore, the vanishing of $(A B)^{2}$ is a necessary condition for the possibility of the above construction.

For convenience such a construct will now be termed a $\Phi$-tetrahedron of the two quadrics.

\section{Considerations of duality..}

It can easily be shown that the vertices $P, Q, R$ and $S$ of a. $\Phi$-tetrahedron lie on the quadric $k$, the vanishing of $\Phi$ being the condition for $k$ and $\chi$ to be polar reciprocal quadrics with respect to $f^{\prime}$. For the polar reciprocal of $\chi$ with respect to $f^{\prime}$ is given by 


$$
b^{\prime}{ }_{x}\left(A b b^{\prime}\right)\left(A b b^{\prime \prime}\right) b^{\prime \prime}{ }_{x}=0,
$$

i.e.

$$
\frac{1}{2} \dot{b}_{x}^{\prime}\left(A b b^{\prime}\right)\left(A \dot{b} b^{\prime \prime}\right) b^{\prime \prime}{ }_{x}=0 \text {, }
$$

which by determinantal permutations gives

$$
\frac{1}{2}\left(A b b^{\prime}\right)^{2} b^{\prime \prime}{ }_{x}{ }^{2}-\dot{1}_{2} \dot{a}^{\prime}{ }_{x}\left(A b b^{\prime}\right)\left(\dot{a} b^{\prime \prime} b b^{\prime}\right) b^{\prime \prime}{ }_{x}=0,
$$

reducing to

$$
\frac{1}{2}(A B)^{2} b_{x}^{2}-\frac{1}{3}(A \beta x)^{2}=0,
$$

$$
3 \Phi f^{\prime}-k=0,
$$

and for $\Phi$ zero this gives $k=0$.

Thus, each $\Phi$-tetrahedron has its vertices on $k$, its faces touching $\chi$, its edges belonging to $\Pi$, and is self-conjugate for $\Pi^{\prime}$. The vanishing of $\Phi$ is obviously also a necessary condition for a $\Phi$-tetrahedron to exist with the same relationships to $k^{\prime}, \chi^{\prime}, \Pi^{\prime}$ and $\Pi$.

\section{A further property of $k$ and $\chi$.}

Before the sufficiency of the condition is considered, the following additional property associated with $k$ and $\chi$ will be established as it is required at a later stage:

$$
\begin{aligned}
(A \beta . B u)^{2} & =\left[\dot{a}_{\beta}\left(\dot{a^{\prime} B u}\right)\right]^{2} \\
& =\left[\dot{b}_{\beta}\left(A \dot{b}^{\prime} u\right)\right]^{2}+2(A B)\left(A \dot{b}^{\prime} u\right) \dot{b}_{\beta} u_{\beta}, \quad \text { since }(A B)^{2}=0 \\
& =2 b_{\beta}^{2}\left(A b^{\prime} u\right)^{2}-2 b_{\beta}\left(A b^{\prime} u\right) b_{\beta}{ }_{\beta}(A b u)+4(A B)\left(A b^{\prime} u\right) b_{\beta} u_{\beta} \\
& =2 b_{\beta}^{2}\left(A b^{\prime} u\right)^{2}-\frac{1}{2}_{b^{\prime}}^{\prime 2}(A b u)^{2}-\left(A b^{\prime} u\right)^{2} b_{\beta}^{2} \\
& =\frac{1}{2} \Delta^{\prime} \chi .
\end{aligned}
$$

Hence, if the plane $u$ belongs to $\chi$ (when $\Phi$ is zero), it also satisfies

$$
(A \beta . B u)^{2}=0 .
$$

Therefore, by the Clebsch Transference Principle every plane belonging to $\chi$ cuts $k$ in a conic which is outpolar to the conic section of $f^{\prime}$ by $u$.

\section{Investigation of the sufficiency of the condition $\Phi=0$.}

In the following it will now be assumed that the quadrics $f$ and $f^{\prime}$ are subject only to the condition that the invariant $\Phi$ vanishes. A plane $u$ will cut $f$ in the conic section given by

$$
(A u v)^{2}=0 .
$$


Let the plane $u$ belong to $\chi$. Then the polar reciprocal cone of $(A u v)^{2}$ with respect to $f^{\prime}$ is obtained from the equation

$$
b_{x}(A b u)\left(A b^{\prime} u\right) b^{\prime}=0,
$$

where $\quad b_{x}(A b u)\left(A b^{\prime} u\right) b^{\prime}{ }_{x}=\dot{b}_{x}(A b u)\left(A \dot{b}^{\prime} u\right) b_{x}^{\prime}, \quad$ since $(A b u)^{2}=0$

$$
\begin{aligned}
& =-(u A B x)(u A b) b_{x}^{\prime} \\
& =-\frac{1}{2}(u A B x)^{2} .
\end{aligned}
$$

Let the point $y$ be the pole of $u$ with respect to $f^{\prime}$. From (3.1) $y$ lies on the quadric $k$, and from the foregoing construction is also the vertex of the polar reciprocal cone of the conic $(A u v)^{2}$ with respect to $f^{\prime}$.

Thus,

$$
\begin{aligned}
& (u B A x)^{2}=b_{y}(b B A x)\left(b^{\prime} B A x\right) b^{\prime}{ }_{y} \\
& =2 b_{y}(b B A x)\left(b^{\prime} B a\right) a_{x}^{\prime} b^{\prime}{ }_{y} \\
& =\frac{2}{3} \dot{b}_{y}(b B A x)\left(b^{\prime} \dot{b}_{1} \dot{b}_{2} a\right) a_{x}^{\prime} b^{\prime}{ }_{y} \text {, where } B=b_{1} b_{2} \\
& ={ }_{3}^{2} \dot{b}_{y}^{\prime}(b B A x)(b \dot{B a}) a_{x}^{\prime} b_{y}^{\prime} \\
& =\dot{s}_{3}^{\prime} \dot{b}_{y}^{\prime} a_{\beta} \bar{a}^{\prime} \dot{a}_{\beta} a_{x}^{\prime} b_{y}^{\prime} \\
& =\stackrel{2}{*}(A \beta x) a_{\beta} a_{x}^{\prime}{ }_{b^{\prime}}{ }_{y}^{2}-\frac{2}{3}(A \beta x) a_{y} b^{\prime}{ }_{\beta} a_{x}{ }_{x} b^{\prime}{ }_{y} \\
& =i_{i}^{1}(A \beta x)^{2} b_{y}^{2}-{ }_{i j}^{1} b_{\beta}^{\prime 2} \dot{a}_{x}^{\prime} a_{y} a_{x}^{\prime} \dot{a}_{y} \\
& ={ }_{i}^{1}(A \beta x)^{2} b_{y}^{2}-{ }_{i: 3}^{1} b_{\beta}^{2}(A y x)^{2} \text {. }
\end{aligned}
$$

Also $\quad(u B A x)(B A)=b_{y}(b B A x)(B A)$

$$
\begin{aligned}
& =-2 b_{y}(a b B) a_{x}^{\prime}\left(a a^{\prime} b_{1} b_{2}\right) \\
& =-2_{3}^{2} \dot{b}_{y}(a b B) a_{x}^{\prime}\left(a a^{\prime} \dot{b}_{1} \dot{b}_{2}\right) \\
& =-\frac{2}{3}_{3} \dot{a}_{y}^{\prime} a_{\beta} a_{x}^{\prime} \dot{a}_{\beta} \\
& =-\frac{1}{3}(A \beta y)(A \beta x) .
\end{aligned}
$$

By combining (i), (ii) and (iii) and using the identity

$$
(u A B x) \equiv-(u B A x)-(B . A) u_{x}
$$

we have

$$
\begin{aligned}
(u A B x)^{2} & \equiv(u B A x)^{2}+2(u B A x)(B A) u_{x}, \quad \text { since } \Phi=0 \\
& \equiv{ }_{: 3}^{1}(A \beta x)^{2} b_{y}^{2}-{ }_{1}^{1} b_{\beta}^{2}(A y x)^{2}-{ }_{3}^{2}(A \beta y)(A \beta x) b_{y} b_{x} .
\end{aligned}
$$

Thus, the polar reciprocal cone of $(A u v)^{2}$ with respect to $f^{\prime}$, where $u$ is a plane of $\chi$ and $\Phi$ vanishes, is given by the equation 


$$
4 b_{y}^{2}(A \beta x)^{2}-b_{\beta}^{2}(A y x)^{2}-8(A \beta y)(A \beta x) b_{y} b_{x}=0 \quad \text { (x current) }
$$

To construct a $\Phi$-tetrahedron let us take, as in $\$ 2$, a triangle $Q R S$ self-conjugate for the section of $f^{\prime}$ by $u$ and circumscribing $(A u v)^{2}$, where $u$ is a plane touching $\chi$, and $P(y)$ is the pole of $u$ with respect to $f^{\prime}$. In order to complete the construction it will be necessary to choose $u$ and the triangle $Q R S$ so that $P Q, P R$ and $P S$ are generators common to the cones $(A y x)^{2}$ and the polar reciprocal of $(A u v)^{2}$ with respect to $f^{\prime}$. This condition is essential since $P Q, P R$ and $P S$ must be lines of $\Pi$ and also the respective polar lines of $R S, S Q$ and $Q R$, which touch $(A u v)^{2}$.

From (5.1) it is seen that the points common to the quadric $k$, the polar reciprocal cone of $(A u v)^{2}$ with respect to $f^{\prime}$ and the plane $u$ (viz. $b_{y} b_{x}=0$ ) also lie on the cone $(A y x)^{2}$. Thus, if a plane $u$ of $\chi$ can be obtained containing a triangle $Q R S$ whose sides touch $(A u v)^{2}$ and whose vertices lie on the section of $k$ by $u$, then since the triangle $Q R S$ is self-conjugate for $f^{\prime}$ and $P(y)$ is the pole of $u$ with respect to $f^{\prime}$, the lines $P Q, P R$ and $P S$ will be generators of the polar reciprocal cone of $(A u v)^{2}$ with respect to $f^{\prime}$ and also generators of $(A y x)^{2}$.

The problem of determining a $\Phi$-tetrahedron is thus reduced from a problem in [3] to one in [2], viz. to obtain the planes $u$ touching $\chi$, which have a triangle $Q R S$ circumscribing the conic $(A u v)^{2}$, inscribed in the section of $k$ by $u$ and self-conjugate for the section of $f^{\prime}$ by $u$.

\section{Lemma concerning a configuration of three conics in a plane.}

To solve the problem outlined at the end of $\$ 5$ the following three ternary forms and their duals will be considered:-

$$
f_{1} \equiv a_{x}^{2}, \Sigma_{1} \equiv u_{\alpha}^{2} ; f_{2} \equiv b_{x}^{2}, \Sigma_{2} \equiv u_{\beta}^{2} ; f_{3} \equiv c_{x}^{2}, \Sigma_{3} \equiv u_{\gamma}^{2} .
$$

It is to be noted that the symbols $a, \alpha ; b, \beta ; c, \gamma$ here used are in no wise related to the corresponding symbols used in connection with the quaternary forms.

The following identities connecting the non-symbolical and symbolical forms of the concomitants of two conics will be found necessary, and can easily be verified:

$$
\begin{aligned}
& f \equiv \sum_{i, j=1}^{3} a_{i j} x_{i} x_{j} \equiv a_{x}^{2}, \\
& \Sigma \equiv \sum_{i, j=1}^{3} A_{i j} u_{i} u_{j} \equiv{ }_{2}^{1} u_{a}^{2},
\end{aligned}
$$




$$
\begin{aligned}
\Delta & \equiv\left|\begin{array}{lll}
a_{11} & a_{12} & a_{13} \\
a_{21} & a_{22} & a_{23} \\
a_{31} & a_{32} & a_{33}
\end{array}\right| \equiv \frac{1}{6} a_{a}^{2}, \\
\Theta & \equiv \sum_{i, j=1}^{3} b_{i j} A_{i j} \equiv \frac{1}{2} b_{a}^{2} .
\end{aligned}
$$

Necessary and sufficient conditions for the existence of a triangle circumscribed about the conic $f_{1}$, inscribed in $f_{2}$ and self-conjugate for $f_{3}$ will now be determined.

Obviously, the following conditions will be necessary:-

$$
\begin{aligned}
& b_{\gamma}^{2}=0, \text { since } f_{2} \text { is outpolar to } \Sigma_{3}, \\
& c_{a}^{2}=0, \text { since } \Sigma_{1} \text { is inpolar to } f_{3} .
\end{aligned}
$$

There is a third necessary condition, however, arising from the fact that a triangle is inscribed in $f_{2}$ and circumscribed about $f_{1}$. In nonsymbolical form the necessary (and sufficient) condition for this last construction is $\Theta^{2}=4 \Delta \Theta^{\prime}$. $^{1}$

From (6.1) the symbolical form of this third necessary condition is therefore

$$
b_{\alpha}^{2} b_{\alpha}^{\prime 2}={ }_{;}^{4} a_{\alpha}^{2} a_{\beta}^{\prime 2}
$$

which by the interchange of equivalent symbols reduces to the slightly modified form

$$
b_{a}^{2} b_{a^{\prime}}^{\prime 2}=\left(a a^{\prime} \beta\right)^{2} \text {. }
$$

It can easily be shown that the condition $(6.4(a))$ also covers the case where $\Sigma_{1}$ degenerates to a point pair.

It will now be proved that conditions (6.2), (6.3) and (6.4) are also sufficient for the possibility of the above construction, and yield a unique triangle satisfying the conditions.

The configuration now to be considered consists of the conic envelope $\Sigma_{1}$, and conic loci $f_{2}$ and $f_{3}$ subject to conditions (6.2), (6.3) and $(6.4(\mathrm{a}))$. Since the envelope $\Sigma_{1}$ is taken as fundamental, its point form becomes $S_{1} \equiv\left(\alpha \alpha^{\prime} x\right)^{2}$, where $\left(a a^{\prime} x\right)^{2} \equiv \frac{4}{3} a_{a}^{2} f_{1}$, and $\left(\alpha a^{\prime} \beta\right)^{2}$ is the $\Theta^{\prime}$ invariant of $S_{1}$ and $\Sigma_{2}$. Also, since $b_{\gamma}^{2}$ is zero, the polar reciprocal of $f_{2}$ with respect to $f_{3}$ is their $\Phi$-conic, ${ }^{2}$ viz.

$$
\Phi_{23} \equiv(b c u)^{2}=0 \text {. }
$$

1 (4) Sommerville, Analytical Conics, p. $279, \$ 23$.

2 (4) p. 286, Ex. 2, but the result can easily be proved symbolically. 
There are four tangents common to the conic envelopes $\Sigma_{1}$ and $\Phi_{23}$, and these determine the range of conic envelopes

$$
\Sigma_{\lambda} \equiv(b c u)^{2}+\lambda u_{a}^{2}=0 .
$$

It can be shown by applying the Clebsch Transference Principle to the binary domain that the point equation of $\Sigma$, is

$$
S_{\lambda} \equiv\left(b c . b^{\prime} c^{\prime} \cdot x\right)^{2}+2 \lambda(b c . a . x)^{2}+\lambda^{2}\left(a a^{\prime} x\right)^{2}=0 .
$$

The $\Theta^{\prime}$-invariant of $S_{2}$ and $\Sigma_{2}$ is therefore

$$
\left(b c . b^{\prime} c^{\prime} . \beta\right)^{2}+2 \lambda(b c . \alpha . \beta)^{2}+\lambda^{2}\left(\alpha a^{\prime} \beta\right)^{2},
$$

and so for a triangle to be inscribed in $f_{2}$ and circumscribed about $\Sigma_{\chi}$ the following necessary and sufficient condition of type $(6.4(a))$ will have to be proved:-

$$
\begin{aligned}
{\left[\left(b c b^{\prime}\right)^{2}+\lambda b_{a}^{2}\right]\left[\left(b^{\prime \prime} c^{\prime} b^{\prime \prime \prime}\right)^{2}+\lambda b_{a^{\prime}}^{\prime 2}\right]=\left(b c . b^{\prime} c^{\prime} \cdot \beta\right)^{2}+2 \lambda(b c . a . \beta)^{2} } \\
+\lambda^{2}\left(a a^{\prime} \beta\right)^{2}
\end{aligned}
$$

where $b, b^{\prime \prime} ; c, c^{\prime} ; a, a^{\prime}$ etc. are equivalent pairs of symbols.

The left-hand side of the above equation reduces to

$$
\left(c_{\beta}^{2}+\lambda b_{a}^{2}\right)\left(c_{\beta^{\prime}}^{\prime 2}+\lambda b_{a^{\prime}}^{\prime 2}\right)=c_{\beta}^{2}{c^{\prime}}_{\beta^{\prime}}^{2}+2 \lambda b_{a}^{2} c_{\beta}^{2}+\lambda^{2} b_{a}^{2} b_{a^{\prime}}^{\prime 2},
$$

while the right-hand side becomes

$$
\begin{aligned}
& {\left[\left(b b^{\prime} c^{\prime}\right) c_{\beta}-\left(c b^{\prime} c^{\prime}\right) b_{\beta}\right]^{2}+2 \lambda\left[b_{a} c_{\beta}-c_{a} b_{\beta}\right]^{2}+{ }_{{ }_{3}^{4}}^{4} \lambda^{2} a_{a}^{2} a_{\beta}^{\prime 2}} \\
& =c_{\beta}^{2}{c^{\prime}}^{\prime 2}+b_{\beta}^{2} b^{\prime 2}{ }_{\gamma}-2 c_{\beta} b_{\beta}\left(b b^{\prime} c^{\prime}\right)\left(c b^{\prime} c^{\prime}\right)+2 \lambda\left(b_{a}^{2} c_{\beta}^{2}+c_{\alpha}^{2} b_{\beta}^{2}-2 b_{\alpha} c_{\beta} b_{\beta} c_{\alpha}\right) \\
& +{ }_{-3}^{4} \lambda^{2} a_{a}^{2} a_{\beta}^{\prime 2}
\end{aligned}
$$

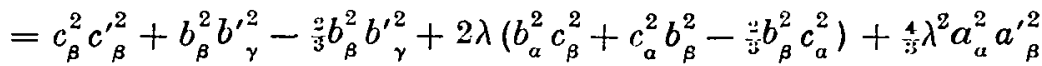

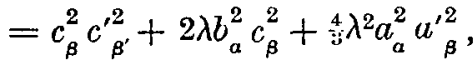

since relations $(6.2)$ and $(6.3)$ are satisfied by the three conics $f_{1}, f_{2}$. and $f_{3}$.

Also, from (6.4)

$$
b_{a}^{2}{b^{\prime}}_{a^{\prime}}^{2}=\frac{4}{3} a_{a}^{2} a_{\beta}^{\prime 2} \text {. }
$$

Thus, (6.5) is established, and so for all values of $\lambda$ an infinity of triangles can be inscribed in $f_{2}$ and circumscribed about $\Sigma_{\lambda}$.

Now let the four tangents common to $\Sigma_{1}$ and $\Phi_{23}$ meet in the point pairs $A, A^{\prime} ; B, B^{\prime}$; and $C, C^{\prime}$, which are three degenerate conic envelopes of the range $\Sigma_{\lambda}$. By application of (6.5) to the point pair $A, A^{\prime}$ a triangle can therefore be inscribed in $f_{2}$ and circumscribed about the degenerate conic envelope whose tangent lines pass through $A, A^{\prime}$. Thus, either $A$ or $A^{\prime}$ must lie on $f_{2}$, and similarly, $B$ or $B^{\prime}$ and $C$ or $C^{\prime}$ must also lie on $f_{2}$. As it is impossible for three collinear 
points to lie on a conic, there is no loss of generality in taking the points $A, B$ and $C$ as the three points on $f_{2}$. Therefore, the triangle $A B C$ is inscribed in $f_{2}$ and circumscribed about $\Phi_{23}$ and $\Sigma_{1}$. Mloreover, since $\Phi_{23}$ is the polar reciprocal of $f_{2}$ with respect to $f_{3}$ and $f_{2}$ is outpolar to $f_{3}$, this triangle is also self-conjugate for $f_{3}$.

The triangle $A B C$ is therefore a unique triangle eircumscribing $\Sigma_{1}$, inscribed in $f_{2}$ and self-conjugate for $f_{3}$, provided (6.2), (6.3) and (6.4) are satisfied.

7. Application to the $\Phi$-tetrahedron of lwo quadrics.

In the case of the three-dimensional configuration it was shown in $\$ 5$ that in order to construct a $\Phi$-tetrahedron, when $\Phi$ vanishes, it is necessary to obtain a plane $u$ belonging to $\chi$, having a triangle circumscribing $(A u v)^{2}$ inscribed in the section of $k$ by $u$, and selfconjugate for the section of $f^{\prime}$ by $u$.

From (4.1), provided $u$ belongs to $\chi$, then $(A u v)^{2}$ is inpolar to $f^{\prime}$, and the section of $k$ by $u$ is outpolar to the section of $f^{\prime}$ by $u$. Thus, if $(A u v)^{2}$ is taken to correspond to $\Sigma_{1}$, the section of $k$ by $u$ to correspond to $f_{2}$, and the section of $f^{\prime}$ by $u$ to correspond to $f_{3}$, where $\Sigma_{1}, f_{2}$ and $f_{3}$ represent the conics dealt with in $\S 6$, then (6.2) and (6.3) are satisfied by the conic sections of $f, k$ and $f^{\prime}$ by $u$. By the application of the Clebsch Transference Principle to relation (6.4) will be found the $\infty^{2}$ planes $u$, which are such that a triangle can be inscribed in the section of $k$ by $u$, and circumscribed about the section of $f$ by $u$. This condition is therefore

$$
\left[\left(A \beta . A^{\prime} u\right)^{2}\right]\left[\left(A^{\prime \prime} \beta^{\prime} . A^{\prime \prime \prime} u\right)^{2}\right]=4_{3}^{4} u_{a}^{2}\left(a . A \beta . A^{\prime} \beta^{\prime} . u\right)^{2},
$$

which represents a surface of class four. The $\infty^{1}$ planes common to this surface and the quadric envelope $\chi$ therefore produce conic sections which satisfy conditions (6.2), (6.3) and (6.4) simultaneously, and so constitute the developable surface of class eight whose planes are the faces of one system of $\Phi$-tetrahedra, viz. that system whose edges touch $f$, and whose members are self-conjugate for $f^{\prime}$. Dually, the $\infty^{1}$ vertices of this system will describe an octavic curve as locus.

Expressed in terms of the irreducible concomitants of two quadrics the developable is given by the equations

$$
\chi=0 \text { and } \Delta \Sigma^{\prime 2}-16 \Theta^{\prime} \Sigma \Sigma^{\prime}+12 \Delta^{\prime} \Sigma \chi^{\prime}=0 .
$$

and the curve by the equations

$$
k=0 \text { and } \Delta f^{\prime 2}-16 \Theta f f^{\prime} \div 8 f k^{\prime}=0 .
$$


The reduction of (7.1) and its dual to forms (7.2) and (7.3) is rather long and involved, and consequently the detailed proof is included as an Appendix.

Similarly, for the second system of $\Phi$-tetrahedra whose edges touch $f^{\prime}$ and whose members are self-conjugate for $f$, the developable and curve are given respectively by the equations

$$
\begin{array}{lll}
\chi^{\prime}=0 & \text { and } & \Delta^{\prime} \Sigma^{2}-16 \Theta \Sigma \Sigma^{\prime}+12 \Delta \Sigma^{\prime} \chi=0, \\
k^{\prime}=0 & \text { and } & \Delta^{\prime} f^{2}-16 \Theta^{\prime} f f^{\prime}+8 f^{\prime} k=0 .
\end{array}
$$

\section{Conclusion.}

Certain aspects of the foregoing theory can be applied to the case where $\Phi$ is the $(A B)^{2}$ invariant of a quadratic complex $(A p)^{2}$ and a quadric $(B p)^{2}$ with further modifications where a Battaglini complex is involved.

In the case of the $\Phi$-invariant of two general quadratic complexes, $(A p)^{2}$ and $(B p)^{2}$, an entirely different method of approach is necessary.

These considerations are deferred meantime.

\section{Appendix.}

The following is the detailed working involved in deriving relations (7.2)-(7.5) from equation (7.1), viz.

$$
\left(A \beta . A^{\prime} u\right)^{2}\left(A^{\prime \prime} \beta^{\prime} . A^{\prime \prime \prime} u\right)^{2}={ }_{3}^{4} u_{a}^{2}\left(a . A \beta . A^{\prime} \beta^{\prime} \cdot u\right)^{2} \text {. }
$$

Firstly, $\left(A \beta . A^{\prime} u\right)^{2}=\left[\dot{a}_{\beta}\left(\dot{a}^{\prime} A^{\prime} u\right)\right]^{2}$

$$
\begin{aligned}
& =2 a_{\beta}^{2} u_{\alpha}^{2}-2 a_{\beta} a_{\beta}^{\prime}\left(a A^{\prime} u\right)\left(a^{\prime} A^{\prime} u\right) \\
& =2 a_{\beta}^{2} u_{\alpha}^{2}-\dot{a}_{\beta} a_{\beta}^{\prime}\left(a A^{\prime} u\right)\left(a^{\prime} \dot{a}_{1} a_{2} u\right), \text { where } A^{\prime} \equiv a, a_{2}, \\
& =2 a_{\beta}^{2} u_{\alpha}^{2}-a_{\beta}^{\prime 2} u_{\alpha}^{2}+z_{\beta}^{2} u_{\beta} a_{\beta} u_{\alpha} a_{a}^{\prime} \\
& ={ }_{i}^{4} a_{\beta}^{2} u_{\alpha}^{2}+{ }_{6}^{3} a_{a}^{2} u_{\beta}^{2} .
\end{aligned}
$$

(A.1) Thus, $\left(A \beta . A^{\prime} u\right)^{2}\left(A^{\prime \prime} \beta^{\prime} . A^{\prime \prime \prime} u\right)^{2}={ }_{3}^{\prime \prime} a_{\beta}^{2} a_{\beta^{\prime}}^{\prime 2} u_{\alpha}^{2} u_{\alpha^{\prime}}^{2}+{ }_{3 ;}^{1} a_{a}^{2} a_{a^{\prime}}^{\prime 2} u_{\beta}^{2} u_{\beta^{\prime}}^{2}$

Secondly, $+{ }_{y}^{4} a_{\alpha}^{2} a_{\beta}^{\prime 2} u_{a^{\prime}}^{2} u_{\beta^{\prime}}^{2}$

where

$$
\text { (a. } \left.A \beta \cdot A^{\prime} \beta^{\prime} . u\right)^{2}=\left[\dot{a}_{1 \beta_{1}} \bar{a}_{2 \beta_{2}}\left(\dot{a_{1}} \bar{a}_{2}^{\prime} a u\right)\right]^{2},
$$

This reduces to

$$
A \beta \equiv \dot{a}_{1 \beta_{1}} \dot{a}_{1}^{\prime} \text { and } A^{\prime} \beta^{\prime} \equiv \bar{a}_{2 \beta_{2}} \bar{a}_{2}^{\prime} \text {. }
$$

$\left[a_{1 \beta_{1}} a_{2 \beta_{2}}\left(a^{\prime}{ }_{1} a_{2}^{\prime} a u\right)-a_{{ }_{1} \beta_{1}}^{\prime} a_{2 \beta_{2}}\left(a_{1} a_{2}^{\prime} a u\right)+a_{{ }_{1 \beta_{1}}} a^{\prime}{ }_{2 \beta_{2}}\left(a_{1} a_{2}(u u)-a_{1 \beta_{1}} a_{2 \beta_{2}}^{\prime}\left(a^{\prime}{ }_{1} a_{2} a u\right)\right]^{2}\right.$ 


$$
\begin{aligned}
& =4 a_{\beta}^{2} a_{\beta^{\prime}}^{\prime 2} u_{\alpha}^{2}-2 a_{1 \beta_{1}} a_{1 \beta_{1}}^{\prime} a_{2 \beta_{2}}^{2}\left(a_{1}^{\prime} a_{2}^{\prime} a u\right)\left(a_{1} a_{2}^{\prime} a u\right) \\
& +2 a_{1 \beta_{1}} a_{1 \beta_{1}}^{\prime} a_{2 \beta_{2}} a_{2 \beta_{2}=}^{\prime}\left(a_{1}^{\prime} a_{2}^{\prime} a u\right)\left(a_{1} a_{2} a u\right) \\
& -2 a^{2}{ }_{1 \beta_{1}} a_{2 \beta_{2}} a_{2 \beta_{2}}^{\prime}\left(a_{1}^{\prime} a_{2}^{\prime} a u\right)\left(a_{1}^{\prime} a_{2} a u\right)-2 a_{1_{\beta 1}^{\prime}}^{2} a_{2 \beta_{2}} a_{2 \beta_{2}}^{\prime}\left(a_{1} a_{2}^{\prime} a u\right)\left(a_{1} a_{2} a u\right) \\
& +2 a_{1 \beta_{1}} a_{1 \beta_{1}}^{\prime} a_{2 \beta_{2}} a_{2 \beta_{2}}^{\prime}\left(a_{1} a_{2}^{\prime} a u\right)\left(a_{1}^{\prime} a_{2} a u\right)-2 a_{1 \beta_{1}} a_{1 \beta_{1}}^{\prime} a_{2 \beta_{2}}^{\prime 2}\left(a_{1} a_{2} a u\right)\left(a_{1}^{\prime} a_{2} a u\right) \text {. }
\end{aligned}
$$

In the above expression, by interchanging equivalent symbols $a_{1}$ with $a_{2}$ and $a_{1}^{\prime}$ with $a_{2}^{\prime}$ in the fourth term; $a_{1}^{\prime}$ with $a_{2}$ and $a_{2}^{\prime}$ with $a_{1}$ in the fifth term; $a_{1}$ with $a_{1}^{\prime}$ in the sixth term; and $a_{2}$ with $a_{2}^{\prime}$ in the seventh. term, it may be shown that

$$
\begin{aligned}
\left(a . A \beta \cdot A^{\prime} \beta^{\prime} \cdot u\right)^{2}= & 4 a_{\beta}^{2} a_{\beta^{\prime}}^{\prime 2} u_{\alpha}^{2}-8 a_{1 \beta_{1}} a_{{ }_{1 \beta_{1}}}\left(a_{1_{1}} a_{2}^{\prime} a u\right)\left(a_{1} a_{2}^{\prime} a u\right) a_{2 \beta_{2}}^{2} \\
& +4 a_{1 \beta_{1}} a_{1 \beta_{1}}^{\prime} a_{2 \beta_{2}} a_{2 \beta_{2}}^{\prime}\left(a_{1_{1}^{\prime}} a_{2}^{\prime} a u\right)\left(a_{1} a_{2} a u\right) \\
= & 4(X-2 Y+Z),
\end{aligned}
$$

where $X=a_{\beta}^{2} a_{\beta^{\prime}}^{\prime 2} u_{\alpha}^{2}$,

$$
\begin{aligned}
& Y=a_{1 \beta_{1}} a_{1_{\beta 1}}^{\prime}\left(a^{\prime}{ }_{1} a_{2}^{\prime} a u\right)\left(a_{1} a_{2}^{\prime} a u\right) a_{2 \beta, 2}^{2}
\end{aligned}
$$

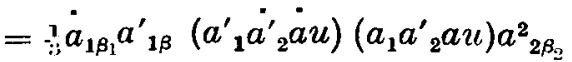

$$
\begin{aligned}
& =\frac{1}{4 ;} a^{\prime 2}{ }_{1 \beta_{1}} a_{2 \beta_{2}}^{2} u_{\alpha}^{2}-\frac{1}{3} u_{\beta_{1}} a_{1 \beta_{1}}^{\prime} a_{1 \alpha}^{\prime} u_{\alpha} a_{2 \beta_{2}}^{2} \\
& =\frac{1}{3} a_{\beta}^{2} a_{\beta^{\prime}}^{\prime 2} u_{\alpha}^{2}-\frac{1}{I_{2}} a_{\alpha}^{2} a_{\beta}^{\prime 2} u_{\beta^{\prime}}^{2} \text {, } \\
& Z=a_{1 \beta_{1}} a_{1 \beta_{1}}^{\prime} a_{2 \beta_{2}} a_{2 \beta_{2}}^{\prime}\left(a^{\prime}{ }_{1} a_{2}^{\prime} a u\right)\left(a_{1} a_{2} a u\right) \\
& =\frac{1}{\bar{b}} a_{1 \beta_{1}} \dot{a}_{1 \beta_{1}}^{\prime} a_{2 \beta_{2}} \dot{a}_{2 \beta_{I}}^{\prime}\left(a_{1}^{\prime} a_{2}^{\prime} a u\right)\left(a_{1} a_{2} \dot{a} u\right) \\
& ={ }_{6}^{1}\left[a_{1 \beta_{1}} \dot{a}_{1 \beta_{1}} a_{2 \beta_{2}} \dot{a}_{2 \beta_{2}} u_{\alpha}^{2}-a_{1 \beta_{1}} \dot{u}_{\beta_{1}} a_{2 \beta_{0}} \dot{a}_{2 \beta_{2}} u_{a} a_{1 \alpha}+a_{1 \beta_{1}} \dot{u}_{\beta_{1}} a_{2 \beta_{2}} \dot{a}_{1 \beta_{2}} u_{a} a_{2 a}\right] \\
& =\frac{1}{8}\left[a^{2}{ }_{1 \beta_{1}} a_{2 \beta_{2}}^{2} u_{\alpha}^{2}-a_{1 \beta_{1}} a_{2 \beta_{1}} a_{2 \beta_{2}} a_{1 \beta_{2}} u_{\alpha}^{2}-a_{1 \beta_{1}} u_{\beta_{1}} a_{2 \beta_{2}}^{2} u_{\alpha} a_{1 a}\right. \\
& \left.+a_{1 \beta_{1}} a_{2 \beta_{1}} a_{2 \beta_{2}} u_{\beta_{2}} u_{\alpha} a_{1 \alpha}+a_{1 \beta_{1}} u_{\beta_{1}} a_{2 \beta_{2}} a_{1 \beta_{2}} u_{\alpha} a_{2 \alpha}-a_{1 \beta_{1}}^{2} a_{2 \beta_{2}} u_{\beta_{2}} u_{\alpha} a_{2 \alpha}\right] \text {. }
\end{aligned}
$$

The above six terms of $Z$ are reduced as follows:-

lst term $=a_{\beta}^{2} a_{\beta^{\prime}}^{2} u_{\alpha}^{2}$.

2nd term $=a_{1 \beta_{1}} a_{2 \beta_{1}} a_{2 \beta_{2}} a_{1 \beta_{2}} u_{\alpha}^{2}$

$$
\begin{aligned}
& =a^{2}{ }_{1 \beta_{1}} a_{2 \beta_{2}}^{2} u_{\alpha}^{2}+a_{1 \beta_{1}} \dot{a}_{2_{\beta_{1}}} a_{2 \beta_{2}} \dot{a}_{1 \beta_{2}} u_{\alpha}^{2} \\
& =a_{1 \beta_{1}}^{2} a_{2 \beta_{2}}^{2} u_{\alpha}^{2}+\left(a_{1} b_{1} b_{2} b_{3}\right)\left(a_{2} a_{1} \dot{b}_{2} \dot{b}_{3}\right) a_{2 \beta_{2}} b_{1 \beta_{2}} u_{\alpha}^{2} \text {, where } \beta_{1} \equiv b_{1} b_{2} b_{3} \text {. } \\
& =a^{2}{ }_{1 \beta_{1}} a^{2}{ }_{2 \beta_{2}} u_{a}^{2}+3{ }_{4}^{3}\left(a_{1} \dot{b}_{1} b_{2} b_{3}\right)\left(a_{2} a_{1} b_{2} b_{3}\right) a_{2 \beta_{2}} b_{1 \beta_{2}} u_{a}^{2} \\
& =a^{2}{ }_{1 \beta_{1}} a^{2}{ }_{2 \beta_{2}}{ }_{2} \iota_{\alpha}^{2}-\frac{3}{4}(A B)^{2} b^{2}{ }_{1 \beta_{2}} u_{a}^{2} \\
& =a_{\beta}^{2} a_{\beta^{\prime}}^{\prime 2} u_{a}^{2}, \text { since }(A B)^{2}=0 \text {. }
\end{aligned}
$$


3rd term $=a_{1 \beta_{1}} u_{\beta_{1}} a^{2}{ }_{2 \beta_{2}} u_{\mathrm{a}} a_{1 n}$

$$
\begin{aligned}
& =\frac{1}{4} u_{\beta 1}^{2} a_{2 \beta 2}^{2} a^{2}{ }_{1 a} \\
& ={ }_{4} a_{a}^{2} a_{\beta}^{\prime 2} u_{\beta^{\prime}}^{2} .
\end{aligned}
$$

4th term $=a_{1 \beta_{1}} a_{2 \beta_{1}} a_{2 \beta_{2}} u_{\beta_{2}} u_{a} a_{1 a}$

$$
\begin{aligned}
& =\frac{1}{4} u_{\beta_{1}} a_{2 \beta_{1}} a_{2 \beta_{2}} u_{\beta_{2}} a^{2}{ }_{1 a} \\
& =\frac{1}{4} u_{\beta_{1}}^{2} a_{2 \beta_{2}}^{2} a_{1 a}^{2}+\frac{1}{4} u_{\beta_{1}} \dot{a}_{2 \beta_{1}} a_{2 \beta_{2}} \dot{u}_{\beta_{2}} a^{2}{ }_{1 a} \\
& ={ }_{4}^{1} u_{\beta_{1}}^{2} a_{2 \beta_{2}}^{2} a^{2}{ }_{1 \alpha}+\frac{1}{4}\left(u b_{1} b_{2} b_{3}\right)\left(a_{2} u \dot{b}_{2} \dot{b}_{3}\right) a_{2 \beta_{2}} \dot{b}_{1 \beta_{2}} a^{2}{ }_{1 a} \\
& ={ }_{4}^{1} u_{\beta_{1}} a^{2}{ }_{2 \beta_{2}} a^{2}{ }_{1 a}+{ }_{4}^{3}\left(u b_{1} b_{2} b_{3}\right)\left(a_{2} u b_{2} b_{3}\right) a_{2 \beta_{2}} b_{1 \beta_{2}} a^{2}{ }_{1 a} \\
& =\frac{1}{4} u_{\beta_{1}}^{2} a_{2 \beta_{2}}^{2} a^{2}{ }_{1 a}+\frac{i 3}{1 \delta}\left(u a_{2} b_{2} b_{3}\right)\left(a_{2} u b_{2} b_{3}\right) b^{2}{ }_{1 \beta_{3}} a^{2}{ }_{1 a} \\
& =\frac{1}{4} a_{a}^{2} a_{\beta}^{\prime 2} u_{\beta^{\prime}}^{2}-\frac{3}{I^{6}}\left(B a^{\prime} u\right)^{2} b_{\beta}^{2} a_{\alpha}^{2} \text {. }
\end{aligned}
$$

5th term $=a_{1 \beta_{1}} u_{\beta_{1}} a_{2 \beta_{2}} a_{1 \beta_{2}} u_{a} a_{2 a}$

$$
\begin{aligned}
& ={ }_{4}^{1} a_{1 \beta_{1}} u_{\beta_{1}} u_{\beta_{2}} a_{\beta_{\beta_{2}}} a_{2 a}^{2} \\
& =\frac{1}{4} a_{a}^{2} a_{\beta}^{\prime 2} \dot{u}_{\beta^{\prime}}^{2}-\frac{3}{16}\left(B a^{\prime} u\right)^{2} b_{\beta}^{2} a_{a}^{2} \text { (as for the } 4 \text { th term). }
\end{aligned}
$$

6 th term $=a_{1 \beta_{1}}^{2} a_{2 \beta_{2}} u_{\beta_{2}} u_{\alpha} a_{2 \alpha}$

$$
\begin{aligned}
& =\frac{1}{4} a_{3 \beta_{1}} a_{2 a}^{2} u_{\beta, 2}^{2} \\
& =\frac{1}{4} a_{a}^{2} a_{\beta}^{\prime 2} u_{\beta^{\prime \prime}}^{2}
\end{aligned}
$$

By combining the above six terms, we have $Z=-\frac{1}{1} a_{\alpha}^{2} b_{\beta}^{2}\left(B a^{\prime} u\right)^{2}$.

Thus, from (A.2)

$$
\begin{aligned}
& \frac{4}{3} u_{a}^{2}\left(a \cdot A \beta \cdot A^{\prime} \beta^{\prime} \cdot u\right)^{2}={ }_{-i}^{6} u_{a}^{2}(X-2 Y+Z) \\
& =\frac{16}{3} u_{a}^{2}\left[a_{\beta}^{2} a_{\beta^{\prime}}^{\prime 2} u_{a^{\prime}}^{2}-2\left(\frac{1}{3} a_{\beta}^{2} a_{\beta}^{\prime 2} u_{\alpha^{\prime}}^{2}-\frac{1}{J^{\prime} \cdot} a_{a^{\prime}}^{2} a_{\beta}^{\prime 2} u_{\beta^{\prime}}^{2}\right)\right. \\
& \left.-\gamma_{16}^{1} a_{\alpha^{\prime}}^{2} b_{\beta}^{2}\left(B a^{\prime} u\right)^{2}\right]
\end{aligned}
$$

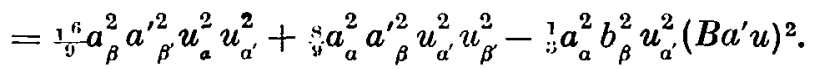

From (A.1) and (A.3) condition (7.1) therefore reduces to

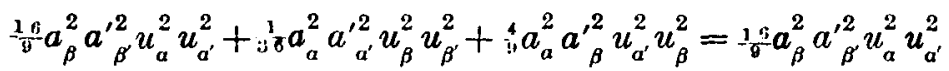

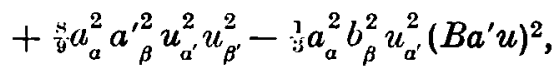

i e.

which gives

$$
a_{a}^{2} a_{a^{\prime}}^{\prime 2} u_{\beta}^{2} u_{\beta^{\prime}}^{2}-16 a_{\alpha}^{2} a_{\beta}^{\prime 2} u_{a^{\prime}}^{2} u_{\beta^{\prime}}^{2}+12 a_{a}^{2} b_{\beta}^{2} u_{\alpha^{\prime}}^{2}\left(B a^{\prime} u\right)^{2}=0
$$

The equations (7.2) determining the developable surface previously considered are therefore

$$
\chi=0 \quad \text { and } \quad \Delta \Sigma^{\prime 2}-16 \Theta^{\prime} \Sigma \Sigma^{\prime}+12 \Delta^{\prime} \Sigma \chi^{\prime}=0 .
$$


To obtain the equations of the curve locus of the vertices of the $\infty^{1} \Phi$-tetrahedra it is merely necessary to reciprocate this developable with respect to $f^{\prime}$, since the vertices are the poles of the faces with respect to $f^{\prime}$.

The equation of the polar reciprocal of the quartic envelope specified in (7.2) is thus given by substituting $b_{x} b$ for $u$ in the latter equation.

The polar reciprocal of $\Sigma$ is given by the following expression equated to zero:

$$
\begin{aligned}
b_{x} b_{a} b_{a}^{\prime} b_{x}^{\prime} & =b_{a}^{2}{b^{\prime 2}}_{x}+\dot{b}_{x} b_{a} \dot{b}_{a}^{\prime} b_{x}^{\prime} \\
& =b_{\alpha}^{2}{b^{\prime}}_{x}^{2}-\frac{1}{2}(B a x)^{2} \\
& =\Theta f^{\prime}-\frac{1}{2} k^{\prime}
\end{aligned}
$$

For $\Sigma^{\prime}$, it is $\quad b_{x} b_{\beta} b_{\beta}^{\prime} b_{x}^{\prime}=\frac{1}{4} b_{\beta}^{2} b^{\prime 2}$

$$
=\frac{1}{4} \Delta^{\prime} f^{\prime}
$$

and for $\chi^{\prime}, b_{x}(B a b)\left(B a b^{\prime}\right) b^{\prime}{ }_{x}=i_{3} \dot{b}_{x}(B a b)\left(B a b^{\prime}\right) b^{\prime}{ }_{x}$

$$
\begin{aligned}
& ={ }_{3}^{1} a_{\beta}^{2} b_{x}^{\prime 2}-{ }_{3}^{1} a_{\beta} b_{\beta}^{\prime} a_{x} b_{x}^{\prime} \\
& =\dddot{1}_{3}^{1} a_{\beta}^{2} b_{x}^{\prime 2}-{ }_{1:}^{1} b_{\beta}^{\prime 2} a_{x}^{2} \\
& ={ }_{3}^{1} \Theta^{\prime} f^{\prime}-{ }_{1: 2}^{1} \Delta^{\prime} f .
\end{aligned}
$$

By substitution of these results in the second equation of (7.2) the quartic surface of the polar reciprocal with respect to $f^{\prime}$ is found to be $\frac{1}{12} \Delta^{2} \Delta^{\prime 2} f^{\prime 2}-4 \Delta \Delta^{\prime} \Theta^{\prime} f^{\prime}\left(\Theta f^{\prime}-\frac{1}{2} k^{\prime}\right)+12 \Delta \Delta^{\prime}\left(\Theta f^{\prime}-\frac{1}{2} k^{\prime}\right)\left({ }_{3}^{1} \Theta^{\prime} f^{\prime}-{ }_{1}^{1}=\Delta^{\prime} f\right)=0$, which reduces to $\Delta f^{\prime 2}-16 \Theta f f^{\prime}+8 f k^{\prime}=0$, and so the equations determining the locus of vertices are, as in (7.3),

$$
k=0 \text { and } \Delta f^{\prime 2}-16 \Theta f f^{\prime}+8 f k^{\prime}=0 .
$$

For the second system of $\Phi$-tetrahedra, (7.4) and (7.5) are true in similar manner.

Heriot-Watt College,

EDinburgh. 